\section{Ajustes nos movimentos de alcançar e apreender objetos: impacto da Síndrome de Down}

\section{Adjustments in the movements of reaching for and grasping objects: the impact of Down Syndrome}

Mariana Martins dos Santos 1

Ana Carolina de Campos 2

Nelci Adriana Cicuto Ferreira Rocha 3
1-3 Departamento de Fisioterapia. Universidade Federal de São Carlos. Rodovia Washington Luís, Km 235. São Carlos, SP, Brasil. Caixa Postal 676. CEP: 13.565-905. E-mail: mari.santos.fisio@gmail.com

\begin{abstract}
Objectives: to verify the influence that properties of objects have on the reaching and grasping adjustments made by infants with and without Down syndrome (DS) between four to eight months of age.

Methods: 16 infants, eight typical and eight with $D S$, were evaluated once a month from months 4 to 8 . Four spherical objects (large soft, small soft, large hard and small hard) were offered and the first five valid movements were recorded for analysis of the variables: proximal adjustment (uni- and bimanual), distal adjustments (palm orientation, hand opening) and grasping of the object.

Results: the typical infants displayed greater bimanual adjustment for large objects at six and eight months and those with DS at seven months. As for distal adjustments, typical infants varied their behavior while DS showed predominant use of the oblique position. In general, the typical group had greater success in grasping all hard and small soft objects when compared to the infants with DS.

Conclusions: infants with Down syndrome showed a smaller variety of adjustments, which led to lower success in grasping, possibly due to the intrinsic limitations of DS.
\end{abstract}

Key words Motor skills, Infant, Down syndrome

\section{Resumo}

Objetivos: verificar a influência das propriedades dos objetos nos ajustes realizados por lactentes típicos e com Síndrome de Down (SD) dos quatro aos oito meses de idade ao alcançar e apreender objetos.

Métodos: 16 lactentes, avaliados uma vez ao mês dos 4 aos 8 meses, sendo oito típicos e oito com SD. Quatro objetos esféricos (maleável grande, maleável pequeno, rígido grande e rígido pequeno) foram apresentados, e os cinco primeiros movimentos válidos foram registrados para análise das variáveis: ajuste proximal (uni e bimanual), ajuste distal (orientação da palma, abertura da mão) e apreensão do objeto.

Resultados: os lactentes típicos apresentaram mais ajuste bimanual para objetos grandes aos seis e oito meses e os com SD aos sete meses. Quanto aos ajustes distais, os lactentes típicos variaram seu comportamento enquanto os com SD apresentaram uso predominante da posição oblíqua. Em geral, o grupo típico apresentou maior sucesso na apreensão dos objetos rígidos e maleável pequeno quando comparados aos lactentes com $S D$.

Conclusões: Os lactentes com SD apresentaram menor variedade de ajustes o que levou a um menor sucesso na apreensão, possivelmente devido a restrições intrínsecas da $S D$.

Palavras-chave Habilidades motoras, Lactente, Sindrome de Down 


\section{Introdução}

A aquisição dos movimentos de alcançar e apreender objetos por volta de quatro a cinco meses de vida representa um importante marco do desenvolvimento infantil, ao possibilitar uma maior exploração do ambiente e das capacidades do próprio corpo. A aquisição e refinamento destas habilidades permitem a realização de ações cognitivamente dirigidas sobre os objetos, além de favorecer a aquisição de novas habilidades motoras. ${ }^{1-4}$

Para que um objeto seja alcançado e apreendido, é necessário que o lactente perceba as affordances dos objetos, identificando seu posicionamento e características, 5 assim, durante o movimento de alcance o lactente deve realizar ajustes proximais, caracterizados por alcances uni ou bimanuais, e ajustes distais ao movimento, tais como orientação da mão e abertura dos dedos para que tenha sucesso na tarefa.6,7 Ao analisar o alcance e apreensão de lactentes típicos, alguns autores $6,8,9$ evidenciaram que lactentes tão jovens quanto os de quatro a seis meses apresentam a capacidade de realizar ajustes antecipatórios dos seus movimentos de acordo com o objeto apresentado ilustrando a capacidade dos lactentes de perceber os affordances dos objetos e adotar ações adaptativas à demanda da tarefa. Heineman et al. 10 relata que comportamentos adaptativos em geral emergem aos seis meses de idade, tendo seu pico entre 8 e 15 meses, no entanto pouco se sabe sobre as características dos ajustes de movimentos de alcance frente a diferentes demandas ambientais. Ao verificar como diferentes affordances (tamanho e rigidez dos objetos e condições do organismo) influenciam o comportamento motor de lactentes, Silva et al.11 verificaram que até oito meses de idade o tamanho foi mais relevante do que a rigidez para determinação de ajustes proximais, uma vez que os lactentes apresentaram mais alcances bimanuais e maior abertura da mão para objetos grandes e unimanuais para objetos pequenos. No entanto, lactentes com desordem do desenvolvimento como é o caso da Síndrome de Down (SD), parecem ter mais dificuldade para executar tais ajustes durante o alcance e preensão de objetos com diferentes affordances devido a uma maior exigência e complexidade desta tarefa para estas crianças. 12

Campos et al. 13 ao verificarem os efeitos do tamanho e rigidez dos objetos no alcance e apreensão de lactentes com SD dos quatro aos seis meses, observaram que estes lactentes adotam ajustes espaço-temporais durantes a trajetória, tornando o movimento mais longo, resultando em menor sucesso na apreensão quando comparados a lactentes típicos. No entanto, pouco se conhece sobre as características qualitativas no posicionamento dos membros superiores e mãos durante o desenvolvimento do alcance, e se estas características influenciam a menor frequência de apreensão encontrada nesta população. Sabendo que disfunções precoces no desenvolvimento do alcance e apreensão podem comprometer o desempenho da criança em ações funcionais, e considerando características dos lactentes com SD, como hipotonia, lentidão de resposta e limitação para explorar suas próprias capacidades, 13 torna-se importante compreender o processo de aquisição do alcançar e apreender no primeiro ano de vida destes lactentes.

O esclarecimento desta questão deve proporcionar informações relevantes para a estimulação precoce da atividade em lactentes com SD, a fim de direcionar quais estratégias precisam ser facilitadas durante a intervenção. Assim, o presente estudo teve como objetivo verificar a influência do tamanho e rigidez dos objetos nos ajustes dos movimentos de alcançar e apreender objetos realizados por lactentes típicos e com SD de quatro a oito meses de idade.

\section{Métodos}

Os participantes foram selecionados com base em prontuários das mães em Unidades Básicas de Saúde e por encaminhamento de médicos geneticistas da cidade de São Carlos, SP. Dessa maneira, os responsáveis por 48 lactentes foram convidados a participar do estudo. Em razão de recusa ou desistência dos responsáveis, ou de não atendimento dos critérios de inclusão, 16 lactentes concluíram o estudo, sendo oito lactentes com diagnóstico de síndrome de Down do tipo trissomia do cromossomo 21 (grupo SD) e oito lactentes com desenvolvimento típico (grupo LT) na faixa etária de quatro a seis meses. Os dois grupos possuíam quatro crianças do sexo feminino e quatro crianças do sexo masculino.

Todos os lactentes com SD estavam participando duas vezes na semana de um mesmo programa de atendimento convencional de fisioterapia a fim de garantir maior homogeneidade da amostra. Não foram incluídos no estudo os lactentes com idade gestacional inferior a 37 semanas, com baixo peso ao nascimento (abaixo de $2500 \mathrm{~g}$ ), com pontuação de Apgar inferior a sete no primeiro e quinto minuto de vida, ou que apresentaram outras patologias não associadas à síndrome de Down.

Foram excluídos do estudo os lactentes que obtiveram duas faltas nas avaliações mensais por quaisquer razões, que apresentaram sinais de comprometimento no desenvolvimento neuro- 
sensório-motor (lactentes do grupo típico), que apresentaram alguma intercorrência traumática e/ou infecciosa, ou necessitaram de qualquer procedimento cirúrgico no decorrer do estudo. Em caso de choro dos lactentes, as avaliações foram desconsideradas e nova avaliação agendada, desde que respeitado o período de sete dias antes ou após a data de aniversário da criança. Caso houvesse recorrência de choro em duas avaliações mensais, os lactentes eram excluídos do estudo.

O estudo está de acordo com a Resolução 196/96 do Conselho Nacional de saúde e foi aprovado pelo Comitê de Ética da UFSCar (Parecer no $355 / 2006$ ). Todos os responsáveis assinaram o Termo de Consentimento Livre e Esclarecido autorizando a participação no estudo.

Uma vez ao mês, dos quatro aos oito meses, os lactentes foram posicionados em uma cadeira infantil reclinada a $50^{\circ}, 3,6$ e foram apresentados aos lactentes na linha média, na altura dos ombros e a uma distância correspondente ao comprimento da extremidade superior do lactente por um período de um minuto ou até que a criança realizasse sete movimentos de alcance aos quatro objetos esféricos e atrativos, com propriedades distintas um maleável grande (MG), maleável pequeno (MP) rígido grande $(\mathrm{RG})$ e um rígido pequeno (RP).6,14

As imagens foram registradas por três câmeras de vídeo digitais, e posteriormente abertas no sistema Dvideow15 para análise qualitativa dos alcances, sendo que os primeiros cinco alcances para cada objeto foram considerados, totalizando um universo de 1600 alcances possíveis. A análise foi realizada por um avaliador treinado, que obteve índice de concordância de $89 \%$ em treinamento com outros avaliadores experientes (informações adicionais em Rocha et al.3,6).

$\mathrm{O}$ alcance foi definido como o movimento de um ou ambos os membros superiores em direção ao alvo, até a mão tocá-lo. ${ }^{6}$ Após a determinação do início e final de cada movimento, os alcances foram classificados quanto à presença de apreensão do objeto e foi realizada sua categorização:

- Ajustes proximais: iniciativa de direcionar um (alcance unimanual) ou ambos os membros superiores (alcances bimanuais) ao objeto apresentado. ${ }^{3}$

- Ajustes distais: ajustes realizados pelas mãos e dedos no início do alcance, no final do alcance, caracterizado pelo momento do toque e durante a apreensão. Foram avaliadas as orientações da palma da mão (horizontal, vertical e oblíqua) nos três momentos, e também a abertura dos dedos (mão aberta, semi-aberta, com oponência de polegar e fechada) durante o inicio e final do alcance. ${ }^{3}$

- Sucesso na apreensão: apreensão de todo ou parte do objeto, com uma ou ambas as mãos. ${ }^{3}$

O teste de Mann-Withney foi utilizado para verificação da semelhança entre os grupos quanto a idade gestacional (IG), peso ao nascer, estatura e Apgar no primeiro e quinto minutos. Foi utilizado o teste não-paramétrico qui-quadrado, a cada mês, para a comparação das proporções das categorias em cada variável para cada objeto apresentado. O mesmo teste foi aplicado, aos diferentes objetos, para comparação dos grupos LT e SD quanto à resposta para cada variável. Para todas as análises foi consi-derado um nível de significância de 0,05. O software estatístico SAS 9.1 foi utilizado para as análises.

\section{Resultados}

Como demonstra a Tabela 1, os dois grupos apresentavam características básicas semelhantes o que permitiu a comparação entre os grupos. Foram analisados 974 alcances manuais, sendo 531 realizados pelos lactentes típicos e 443 pelos lactentes com SD. Os resultados serão apresentados nas tabelas que seguem, de acordo com os grupos estudados quanto às variáveis qualitativas intra grupo de quatro a oito meses e também entre grupos de acordo com cada tipo de objeto, independente da idade.

Tabela 1

Características dos grupos de lactentes típicos e com síndrome de Down expressos em média e desvio-padrão.

\begin{tabular}{lccccc}
\hline Grupo & IG (semanas) & Peso ao nascer (g) & Estatura & Apgar 1' & Apgar 5' \\
\hline LT & $39,10 \pm 1,28$ & $3,57 \pm 0,34$ & $49,5 \pm 1,2$ & $9,00 \pm 0,75$ & $9,75 \pm 0,46$ \\
SD & $38,04 \pm 0,93$ & $3,17 \pm 0,93$ & $47,62 \pm 2,32$ & $8,12 \pm 0,64$ & $9,25 \pm 0,46$ \\
$p^{*}$ & 0,179 & 0,05 & 0,061 & 0,059 & 0,132 \\
\hline
\end{tabular}

*Teste Mann Whitney; IG= idade gestacional; LT= lactentes típicos; SD= síndrome de Down. 
$\mathrm{Na}$ Tabela 2 estão ilustrados os resultados do grupo LT dos quatro aos oito meses de idade. Podese verificar que este grupo aos seis e oito meses, apresentou mais alcances bimanuais para os objetos grandes ( $M G$ e RG), quando comparados aos pequenos (MP e RP). Outra característica encontrada nestes lactentes é o predomínio da orientação vertical para os objetos MP, RG e RP e da orientação oblíqua para o MG durante a apreensão aos seis meses. Aos sete meses, por sua vez, um predomínio da orientação vertical para os objetos grandes ( $\mathrm{MG} \mathrm{e}$ RG) e uma reduzida frequência de orientações obliquas para os objetos pequenos (MP e RP).

No grupo típico houve um predomínio de sucesso na apreensão ao longo do quinto, sexto, sétimo e oitavo mês para todos os objetos com exceção do RG que atingiu 50\% de sucesso somente aos oito meses. Observa-se ainda aumento na frequência de apreensão para os objetos pequenos ao longo dos meses.

O grupo SD, como pode ser observado na Tabela 3 , apresentou resultados significativos quanto ao ajuste proximal somente aos sete meses, ao realizar mais ajustes bimanuais para os objetos grandes (MG e RG) e unimanuais para os pequenos (MP e RP). Nesta análise, observa-se que no quinto mês o grupo SD não apresentou nenhuma apreensão do objeto RG e obteve frequência inferior a $50 \%$ para todos os outros objetos. A partir do sexto mês foi observada ocorrência de sucesso na apreensão superior a $50 \%$ para o objeto MG e a partir do sétimo mês, para os objetos MP e RP. Quanto ao objeto RG, os lactentes apresentaram em todos os meses uma frequência de sucesso inferior a $50 \%$.

Quando comparados os grupos, não houve diferença significativa no comportamento dos grupos quanto ao ajuste proximal (Tabela 4). Quanto à abertura da mão no inicio do alcance, embora em ambos os grupos tenha predominado a classificação semiaberta, o grupo SD apresentou menor frequência de mão fechada e com oponência do polegar para o objeto RP do que o grupo LT. Os grupos diferiram seus comportamentos também quanto à orientação da palma no final do alcance para os objetos MG, RG e RP, tendo os lactentes com SD apresentado maior frequência da orientação obliqua e os típicos adotado estratégias variadas. No momento da apreensão, estes lactentes realizaram menor frequência de orientação horizontal para o objeto MP em relação ao grupo LT.

Ao comparar os grupos quanto ao sucesso na apreensão, pode-se verificar que o grupo LT apresentou maior frequência de sucesso para os objetos MP, RG e RP em comparação com o grupo SD (Tabela 4).

\section{Discussão}

Este estudo buscou verificar a influência do tamanho e rigidez dos objetos nos ajustes realizados por latentes típicos e com SD dos quatro aos oito meses de idade ao alcançar e apreender objetos.

Referente ao ajuste proximal foi evidenciado que os dois grupos de lactentes ajustam seus movimentos de acordo com o objeto apresentado, visto que apresentaram maior frequência de alcances bimanuais para objetos grandes quando comparados aos objetos pequenos. No entanto, enquanto os lactentes típicos apresentaram esta estratégia aos seis meses de idade, os lactentes com SD realizaram este tipo de ajuste aos sete meses. Esta diferença temporal entre os grupos condiz com os resultados de Cadoret e Beuter, 16 que relatam um atraso na realização de ajustes em lactentes com SD, no que tange a percepção das propriedades de tamanho dos objetos. Tendo em vista a redução da percepção das informações sensoriais e hipotonia axial observada nesta população, 17 inferimos que este atraso se deva tanto a dificuldades na percepção do tamanho dos objetos, quanto à dificuldade no controle proximal bilateral dos membros superiores destes lactentes, necessários para o ajuste bimanual do alcance.

$\mathrm{O}$ aumento na frequência de alcances bimanuais para os objetos grandes (maleáveis e rígidos) sugere que o tamanho do objeto tem uma influência maior sobre o comportamento de alcançar que a rigidez, dado este condizente com a literatura 11,12 Tal resultado nos permite inferir que os lactentes realizaram ajustes antecipatórios levando as duas mãos em direção aos objetos grandes, embora os lactentes com SD o tenham feito com atraso de um mês.

Quanto aos ajustes distais, a abertura da mão no início do alcance diferiu entre os grupos para o objeto RP. Nesta situação, embora os dois grupos tenham apresentando predominância do posicionamento semi-aberto, o grupo SD apresentou menor variedade de posicionamentos que o grupo LT. Considera-se que o predomínio de mão semi-aberta no início do movimento seja característico desta faixa etária, ${ }^{3}$ representando também um posicionamento de repouso das mãos no início do alcance. A menor variedade de posicionamentos observada parece representar uma característica da SD, possivelmente devido a fatores presentes na síndrome como baixo tônus, dificuldade do controle axial, 17 e repertórios motores menos complexos, ${ }^{18}$ que levam a menor movimentação destes lactentes, podendo gerar esta menor variabilidade na abertura da mão no início do movimento.

A existência de um repertório motor menos 


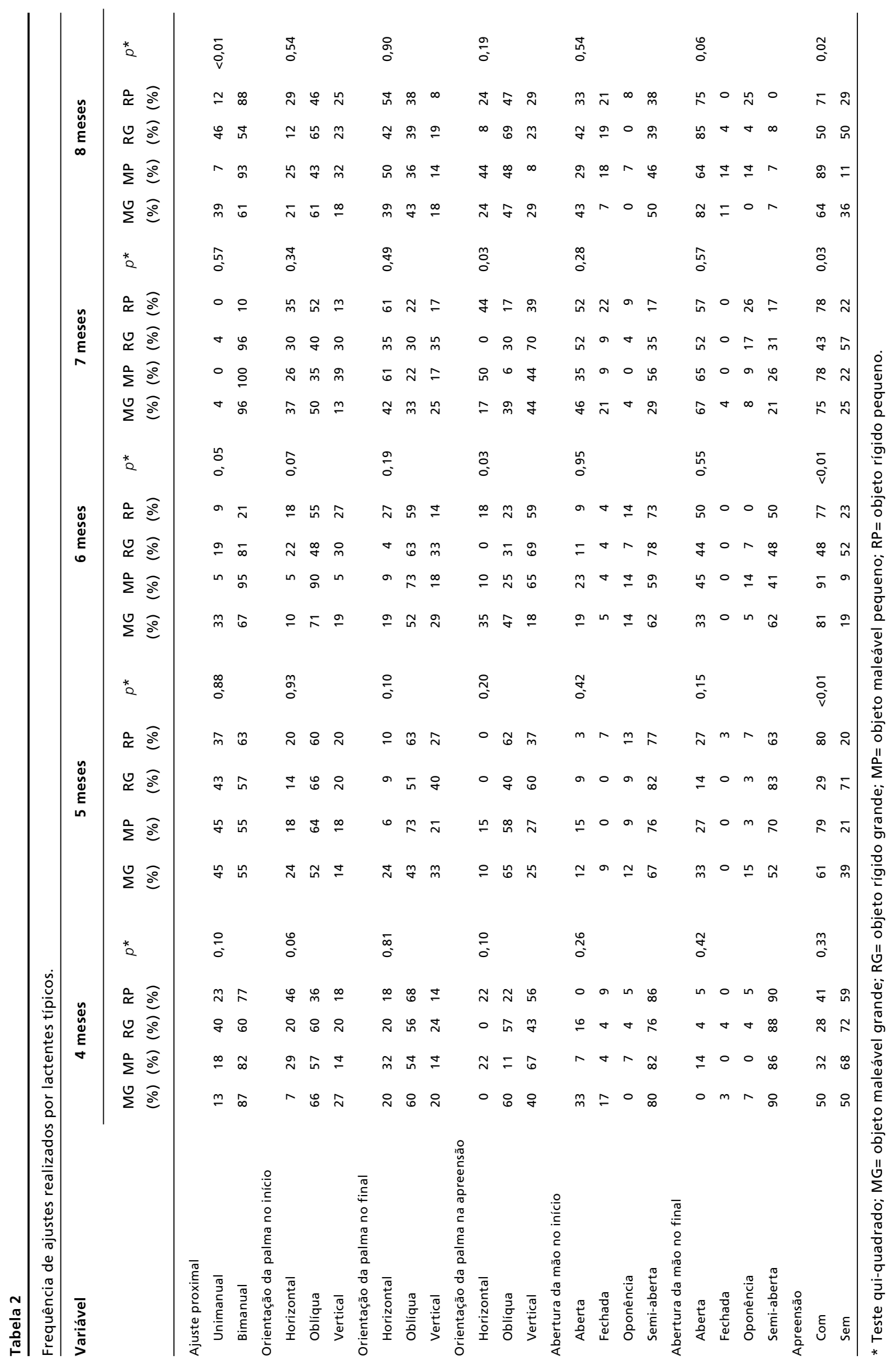




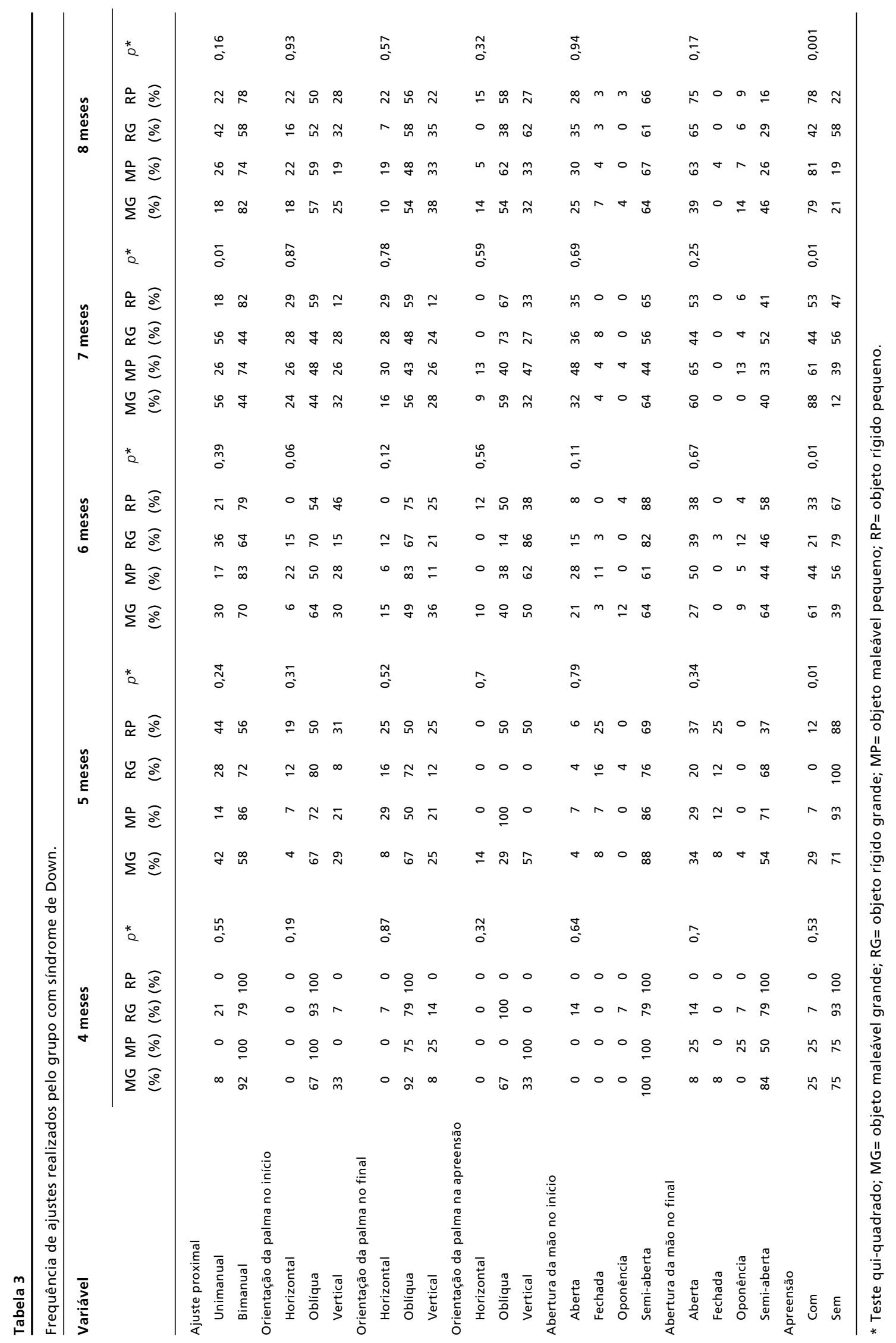


Comparação entre os ajustes realizados pelos grupos lactentes típicos e síndrome de Down, incluindo todos os alcances ao longo de quatro a oito meses, para os diferentes objetos.

\begin{tabular}{|c|c|c|c|c|c|c|c|c|c|c|c|c|}
\hline \multirow[t]{2}{*}{ Variável } & \multicolumn{3}{|c|}{ MG } & \multicolumn{3}{|c|}{ MP } & \multicolumn{3}{|c|}{ RG } & \multicolumn{3}{|c|}{$\mathbf{R P}$} \\
\hline & $\begin{array}{c}\mathrm{LT} \\
(\%)\end{array}$ & $\begin{array}{c}\text { SD } \\
(\%)\end{array}$ & $p^{*}$ & $\begin{array}{c}\mathrm{LT} \\
(\%)\end{array}$ & $\begin{array}{l}\text { SD } \\
(\%)\end{array}$ & $p^{*}$ & $\begin{array}{c}\text { LT } \\
(\%)\end{array}$ & $\begin{array}{r}\text { SD } \\
(\%)\end{array}$ & $p^{*}$ & $\begin{array}{l}\text { LT } \\
(\%)\end{array}$ & $\begin{array}{l}\text { SD } \\
(\%)\end{array}$ & $p^{*}$ \\
\hline \multicolumn{13}{|l|}{ Ajuste proximal } \\
\hline Unimanual & 28 & 33 & 0,39 & 17 & 21 & 0,48 & 32 & 38 & 0,3 & 17 & 24 & 0,22 \\
\hline Bimanual & 72 & 67 & & 83 & 79 & & 68 & 62 & & 83 & 76 & \\
\hline \multicolumn{13}{|c|}{ Orientação da palma no início } \\
\hline Horizontal & 20 & 11 & 0,08 & 21 & 20 & 0,97 & 19 & 16 & 0,4 & 29 & 16 & 0,07 \\
\hline Oblíqua & 60 & 59 & & 57 & 58 & & 57 & 65 & & 50 & 54 & \\
\hline Vertical & 2 & 30 & & 22 & 22 & & 24 & 19 & & 21 & 30 & \\
\hline \multicolumn{13}{|c|}{ Orientação da palma no final } \\
\hline Horizontal & 29 & 11 & $<0,01$ & 31 & 20 & 0,14 & 21 & 14 & 0,1 & 33 & 18 & 0,04 \\
\hline Oblíqua & 46 & 59 & & 52 & 56 & & 49 & 63 & & 50 & 61 & \\
\hline Vertical & 25 & 30 & & 17 & 24 & & 31 & 23 & & 17 & 21 & \\
\hline \multicolumn{13}{|c|}{ Orientação da palma na apreensão } \\
\hline Horizontal & 17 & 11 & 0,37 & 29 & 7 & $<0,01$ & 2 & 0 & 0,7 & 20 & 11 & 0,08 \\
\hline Oblíqua & 52 & 50 & & 35 & 50 & & 45 & 47 & & 38 & 58 & \\
\hline Vertical & 31 & 39 & & 37 & 43 & & 53 & 53 & & 42 & 31 & \\
\hline \multicolumn{13}{|c|}{ Abertura da mão no início } \\
\hline Aberta & 23 & 19 & 0,09 & 21 & 29 & 0,22 & 24 & 22 & 0,4 & 19 & 20 & 0,03 \\
\hline Fechada & 12 & 5 & & 7 & 6 & & 7 & 6 & & 12 & 5 & \\
\hline Oponência & 6 & 4 & & 8 & 1 & & 5 & 2 & & 10 & 2 & \\
\hline Semi-aberta & 59 & 72 & & 65 & 64 & & 64 & 70 & & 59 & 73 & \\
\hline \multicolumn{13}{|c|}{ Abertura da mão no final } \\
\hline Aberta & 42 & 36 & 0,63 & 42 & 54 & 0,29 & 38 & 40 & 0,8 & 42 & 53 & 0,06 \\
\hline Fechada & 4 & 2 & & 3 & 1 & & 1 & 3 & & 0.8 & 4 & \\
\hline Oponência & 7 & 7 & & 7 & 8 & & 7 & 6 & & 12 & 6 & \\
\hline Semi-aberta & 47 & 55 & & 48 & 37 & & 54 & 51 & & 45 & 37 & \\
\hline \multicolumn{13}{|l|}{ Apreensão } \\
\hline Com & 65 & 61 & 0,5 & 73 & 53 & $<0,01$ & 39 & 25 & 0 & 70 & 48 & $<0,01$ \\
\hline Sem & 35 & 39 & & 27 & 47 & & 61 & 75 & & 30 & 52 & \\
\hline
\end{tabular}

* Teste qui-quadrado; $\mathrm{MG}=$ objeto maleável grande; $\mathrm{RG}$ = objeto rígido grande; $\mathrm{MP}=$ objeto maleável pequeno; $\mathrm{RP}=$ objeto rígido pequeno; $\mathrm{LT}=$ lactentes típicos; $\mathrm{SD}=$ síndrome de Down.

variado pode ser um dos fatores pelo qual indivíduos com SD, mesmo na idade adulta, apresentam movimentos caracteristicamente menos eficientes, visto que a exploração de estratégias de movimentos variadas durante o desenvolvimento infantil é pressuposto para a seleção de estratégias motoras adaptativas perante as demandas ambientais. 19

Alguns estudos $3,20,21$ relatam que o aumento do tamanho do objeto deveria resultar em uma maior abertura da mão ao final do alcance, especialmente para os objetos rígidos. No presente estudo, nenhum dos grupos demonstrou influência das propriedades dos objetos para esta variável. Este resultado pode ser explicado pela exigência de maior controle para realizar tal ajuste, que segundo alguns autores20-23 aparece por volta dos nove meses. Apesar de não ter sido um resultado significativo, os lactentes típicos deste estudo, apresentaram aos oito meses, um predomínio de posicionamento de mão aberta no final do alcance para o objeto RG. Referente à orientação da palma da mão no início e final do alcance, os dois grupos de lactentes apresentaram a orientação oblíqua como a mais frequente para todos os objetos na maioria dos meses. No entanto, os resultados nos permitem inferir que enquanto os lactentes típicos utilizaram informações sensoriais para modificar seus posicionamentos de acordo com o objeto, os lactentes com SD continuaram apresentando 
comportamentos semelhantes para todos os objetos. O posicionamento verticalizado ao final do alcance, por exemplo, demonstrou favorecer a apreensão do objeto RG. Além disso, enquanto os LT ajustaram seus movimentos passando de um posicionamento oblíquo ao final do alcance para verticalizado no momento da apreensão a partir do sexto mês, os lactentes com SD mantiveram o posicionamento obliquo em todo o movimento, o que reduziu o sucesso na apreensão deste objeto.

Durante o desenvolvimento típico é esperado que inicialmente os movimentos realizados em uma mesma condição ambiental, sejam mais variáveis e inconsistentes e progridam para movimentos estáveis e eficientes. 24 No entanto, quando a demanda ambiental se modifica, o organismo deve ser capaz de alterar suas estratégias de movimento a fim de obter sucesso na tarefa, apresentado assim, uma adaptação resultante de uma maior variabilidade de movimento.25-27 Em estudo prévio, Campos et al. 27 verificaram que os lactentes com SD apresentaram ajustes espaço-temporais de acordo com o objeto apresentado, sem resultar, no entanto em maior sucesso na apreensão quando comparados a lactentes típicos. No presente estudo, os resultados são indicativos de que os lactentes com SD não estão utilizando estratégias diferentes de orientação da palma no final do alcance e no momento da apreensão. Como consequência, há um empobrecimento da variedade de estratégias disponíveis em seu repertório para a realização de ações adaptadas às demandas ambientais, que resultou em uma menor frequência de apreensão ao longo dos meses. Tais resultados podem ser atribuídos a fatores intrínsecos característicos da SD tais como redução da percepção das informações sensoriais, baixo tônus e resposta motora mais lenta. ${ }^{17}$

Tais resultados nos permitem ressaltar que durante a estimulação dos lactentes com SD deve ocorrer o favorecimento de experiências variadas de movimento com o intuito de promover estratégias mais eficientes de movimentos nestes lactentes, visto que eles apresentam um repertório motor menos variado e menos eficiente para os movimentos de alcançar e apreender objetos com diferentes características. Para tanto, a intervenção deve utilizar a apresentação de objetos rígidos e grandes para favorecer e treinar alcances bimanuais com mão aberta, pequenos para alcances unimanuais e maleáveis para o treino inicial do alcance, visto que o objeto MG se mostrou o mais fácil de apreender.

Os lactentes com SD estudados e nas idades avaliadas não conseguem realizar ajustes de maneira adequada de forma a obter sucesso na tarefa de apreender os objetos, mesmo estando submetidos a um programa convencional de fisioterapia para estimulação do desenvolvimento. Estes lactentes apresentam menor variedade de ajustes de acordo com o objeto apresentado quando comparados a lactentes típicos, o que causou o menor índice de sucesso na apreensão e atraso nas habilidades de ajustes proximais encontrados. Possivelmente tais resultados se devam a presença de restrições intrínsecas características dos lactentes com SD.

\section{Referências}

1. Corbetta D, Snapp-Childs W. The role of sensory-motor experience on the development of infant reaching. Infant Behav Dev. 2009; 32: 44-58.

2. Fagard J. Linked proximal and distal changes in the reaching behavior of 5-to 12-month-old human infants grasping objects of different sizes. Infant Behav Dev. 2000; 23: 317-29.

3. Rocha NACF, Silva FPS, Tudella E. The impact of object size and rigidity on infant reaching. Infant Behav Dev. 2006; 29: 251-61.

4. Thelen E. Motor development: a new synthesis. Am Psychol. 1995; 50: 79-95.

5. Gibson EJ, Pick AP. An ecological approach to perceptual development. In: Gibson EJ, Pick AP, editors. An ecological approach to perceptual learning and development. New York: Oxford University Press; 2000. p. 14-25.

6. Rocha NACF, Silva FPS, Tudella E. Influência do tamanho e da rigidez dos objetos nos ajustes proximais e distais do alcance de lactentes. Rev Bras Fisioter. 2006; 13: 25-30.
7. Lee MH, Liu YT, Newell KM. Longitudinal expressions of infant's prehension as a function of object properties. Infant Behav Dev. 2006; 29: 481-93.

8. Siddiqui A. Object size as a determinant of grasping in infancy. J Genetic Psychol. 1995; 153: 345-58.

9. Newell KM, McDonald PV, Baillargeon R. Body scale and infant grip configurations. Dev Psychobiol. 1993; 26: 195205.

10. Heineman KR, Middelburg KJ, Hadders-Algra M. Ten and twelve month olds visual anticipation of orientation and size during grasping. Acta Paediatr. 2010; 99: 618-24.

11. Silva FPS, Rocha NACF, Tudella E. Tamanho e rigidez dos objetos influenciam os ajustes proximais e distais do alcance de crianças? Rev Bras Fisioter. 2011; 15: 37-44.

12. Toledo AM, Almeida Soares, D, Tudella E. Proximal and adjustments of reaching behaviour in preterms infants. $J$ Mot Behav. 2011; 43: 137-45.

13. Campos AC, Francisco KR, Savelsbergh GJP, Rocha NACF. How do object size and rigidity affect reaching and 
grasping in infants with Down Syndrome? Res Dev Disabil. 2011; 32: 246-52.

14. Campos AC, Rocha NACF, Savelsbergh GJP. Reaching and grasping movements in infants at risk: a review. Res Dev Disabil. 2009; 30: 819-26.

15. Figueroa PJ, Leite NJ, Barros RML. A flexible software for tracking of markers used in human motion analysis. Comput Methods Programs Biomed. 2003; 72: 155-65.

16. Cadoret G, Beuter A. Early development of reaching in Down syndrome infants. Early Hum Dev. 1994; 36: 157-73.

17. Polastri PF, Barela JA. Percepção-ação no desenvolvimento motor de crianças portadoras de síndrome de Down. Rev Sobama. 2002; 7: 1-8

18. McKay SA, Angulo-Barroso RA. Longitudinal assessment of legmotor activity and sleep patterns in infants with and without Down syndrome. Infant Behav Dev. 2006; 29: 15368

19. Hadders-Algra M. Reduced variability in motor behavior: an indicator of impaired cerebral connectivity? Early Hum Dev. 2008; 12: 787-9.

20. Lockman JJ, Ashmead DH, Bushnell EW. The development of anticipatory hand orientation during infancy. J Exp Child Psychol. 1994; 37: 176-86
21. Sacrey LAR, Whishaw IQ. Development of collection precedes targeted reaching: Resting shapes of the hands and digits in 1-6-month-old human infants. Behavioural Brain Research 2010. 214: 125-129.

22. Jakobson LS, Goodale MA. Factors affecting higher-order movement planning: a kinematic analysis of human prehension. Exp Brain Res. 1991; 86: 199-208.

23. Newell KM, Scully DM, McDonald PV, Baillargeon R. Task constraints and infant grip configurations. Dev Psychobiol. 1989; 22: 817-32

24. Thelen E, Corbetta D, Spencer JP. Development of reaching during the first year: Role of movement speed. J Exp Psychol Hum Percept Perform. 1996; 22: 1059-107.

25. Hadders-Algra M. The Neuronal Group Selection Theory: promising principles for understanding and treating developmental motor disorders. Dev Med Child Neurol. 2000; 42: $707-15$

26. Hadders-Algra M. Early brain damage and the development of motor behavior in children clues for therapeutic intervention? Neural Plast. 2001; 1-2: 31-49.

27. Campos AC, Rocha NACF, Savelsbergh GJP. Development of reaching and grasping skills in infants with syndrome de Down. Res Dev Disabil. 2010; 31: 70-80.

Recebido em 12 de setembro de 2011

Versão final apresentada em 23 de fevereiro de 2012

Aprovado em 26 de março de 2012 